\title{
Habilidades gerenciales en mujeres comerciantes cabeza de hogar y economía familiar
}

\author{
Patricia M. Cassab Martínez $z^{1}$ \\ Universidad Pontificia Bolivariana - Sede Montería \\ patricia.cassab@upb.edu.co \\ Dolly Yamile Mayorca Beltrán² \\ Universidad Pontificia Bolivariana - Sede Montería \\ dolly.mayorcab@upb.edu.co
}

DOI: https://doi.org/10.21158/01208160.n84.2018.1922

Fecha de recepción: 31 de agosto de 2017

Fecha de aprobación: 05 de enero de 2018

Cómo citar este artículo / To reference this article / Comment citer cet article / Para citar este artigo:

Cassab Martínez, P. M. y Mayorca Beltrán, D. Y. (2018). Habilidades gerenciales en mujeres comerciantes cabeza de hogar y economía familiar. Revista EAN, 84, (pp 153-166). DOI: https://doi.org/10.21158/01208160.n84.2018.1922

\section{Resumen}

El presente artículo de investigación, describe las habilidades gerenciales que las mujeres comerciantes cabeza de hogar adquieren en sus actividades comerciales por medio de experiencias propias y capacitaciones de las marcas que representan. Tiene como objetivo identificar las habilidades gerenciales y comerciales que desarrollan y su incidencia en la economía familiar; para ello, se aplicó el método descriptivo, analizando el comportamiento de la población sujeta de la investigación. Luego de la tabulación y análisis de datos, los resultados de la investigación muestran las habilidades conceptuales, técnicas y humanas desarrolladas por las mujeres cabeza de hogar en el desempeño de sus actividades comerciales, caracterizadas por la automotivación, liderazgo, buenas relaciones interpersonales y capacitaciones frecuentes.

\section{Palabras clave}

Comercialización, habilidades gerenciales, empoderamiento mujeres, economía familiar, economía de género.

\footnotetext{
${ }^{1}$ Administradora de Empresas - Universidad Autónoma de Bucaramanga, Colombia. Magíster en Innovación para el Desarrollo Empresarial - Instituto Tecnológico de Estudios Superiores de Monterrey, México. Docente Facultad de Administración de Empresas - Escuela de Economía, Administración y Negocios de la Universidad Pontificia Bolivariana Sede Montería y miembro del Grupo de Investigación ESDER, de la misma universidad.

${ }^{2}$ Administradora Industrial - Universidad de Cartagena, Colombia. Magíster en Ciencias de la Organización - Universidad del Valle, Colombia. Docente de la Facultad de Administración de Empresas - Escuela de Economía, Administración y Negocios de la Universidad Pontificia Bolivariana - Sede Montería y miembro del Grupo de Investigación ESDER, de la misma universidad.
} 


\title{
Management skills of trading women as heads of household and in charge of family economy
}

\begin{abstract}
This paper describes all management skills that trading women as heads of household develop when they perform trading activities through own experiences and training of the brand names they represent. It aims at identifying managerial and trading abilities they develop and the influence they have on family economy. For this reason, a descriptive method was used to analyze the behavior of the population studied. After data tabulation and analysis, research outcomes show the theoretical, technical and human skills developed by heads of household women when performing their trading activities, characterized by selfmotivation, leadership, good interpersonal relations and frequent training.
\end{abstract}

Key words: Trading, management skills, women empowerment, family economy, gender economy.

\section{Compétences managériales des femmes commerçantes chefs de famille et économie familiale}

Résumé. Cet article de recherche décrit les compétences managériales acquises par les femmes chefs de famille lors de leurs activités commerciales au travers de leurs propres expériences et grâce aux formations offertes par les marques qu'elles représentent. L'objectif est ici d'identifier les compétences managériales et commerciales acquises et leur impact sur l'économie familiale grâce à une méthode descriptive analysant le comportement de la population de l'enquête. Après compilation et analyse des données, les résultats de l'étude révèlent des compétences conceptuelles, humaines et techniques acquises par ces femmes chefs de famille dans l'exercice de leur activité. Ces femmes se caractérisent de plus par leur leadership, auto-motivation, bonnes relations interpersonnelles et par une formation fréquente.

Mots clefs: Marketing, compétences de gestion, autonomisation des femmes, économie familiale, économie de genre.

\section{Habilidades gerenciais em mulheres comerciantes cabeças de família e economia familiar}

Resumo. O presente artigo de pesquisa descreve as habilidades gerenciais que as mulheres comerciantes cabeças de família adquirem em suas atividades comerciais, por meio de experiências próprias e capacitaçôes das marcas que representam. Tem como objetivo identificar as habilidades gerenciais e comerciais que desenvolvem e sua incidência na economia familiar. Aplicou-se o método descritivo, analisando o comportamento da população sujeito da pesquisa. Depois da tabulação e análise de dados, os resultados da pesquisa mostram as habilidades conceituais, técnicas e humanas desenvolvidas pelas mulheres cabeças de família no desempenho de suas atividades comerciais, caracterizadas pela automotivação, liderança, boas relações interpessoais e capacitações frequentes.

Palavras-chave: Comercialização, habilidades gerenciais, empoderamento mulheres, economia familiar, economia de gênero. 


\section{Introducción}

Tas mujeres comerciantes cabeza de Lhogar, población objeto de este artículo, evidencian la situación que actualmente viven muchas mujeres que cargan sobre sus hombros la responsabilidad de buscar el sostenimiento de sus familias; con el ejercicio de la actividad comercial que ellas realizan, han logrado, no solo conseguir sustento, sino desarrollar habilidades para los negocios, lo cual les ha permitido el mejoramiento de su calidad de vida y el de sus familias y así aportar al desarrollo económico y social de la región y el país.

Colombia, en los últimos años, ha venido mostrando señales de crecimiento económico y avances en los indicadores de desarrollo humano, los cuales se han visto como oportunidades para el progreso social. Sin embargo, toda la población no se ha beneficiado por igual, puesto que las mujeres experimentan brechas en relación con los hombres, en particular, las mujeres cabeza de hogar en la esfera económica. Por tanto, en referencia a los derechos económicos, el acceso de las mujeres al empleo formal y su participación en el mercado laboral, si bien ha ido creciendo, es aún limitado (ONU Mujeres Colombia, s. f.).

En efecto, el Boletín Mujeres en el Mercado Laboral Colombiano, muestra que la tasa de desempleo en las mujeres es del $11.8 \%$ y la de los hombres es del $6.7 \%$, por lo cual el porcentaje de desempleo de las mujeres es un $5.1 \%$ mayor que el de los hombres (Observatorio Servicio Público de Empleo, 2016). En particular, las mujeres jóvenes presentan mayor vulnerabilidad y alcanzan una tasa de desempleo del $20.5 \%$; sumado a esto, las mayores brechas de desempleo entre hombres y mujeres se ubican principalmente en las ciudades de la Costa Caribe, en la cual es Montería la tercera ciudad con mayor brecha en la región con un $2.1 \%$, aquí la tasa de desempleo de hombres es del $5.0 \%$ y de mujeres del $10.4 \%$.

En cuanto a la participación en el mercado laboral, el Boletín Mujeres en el Mercado Laboral Colombiano revela que las mujeres participan menos en el mercado de trabajo, con una proporción de cinco de cada diez mujeres en edad de trabajar, cinco se encuentran trabajando; mientras que, en la relación de hombres, que es de ocho de cada diez en edad de trabajar, ocho son los que se encuentran trabajando.

Con respecto a los sectores, la mayoría de las mujeres se emplean en actividades relacionadas con servicios personales y comercio; es decir, que más de 7.2 millones de mujeres en el país trabajan en servicios comunales, sociales y personales $-67 \%$-, intermediación financiera $-56 \%-$, y comercio, hoteles y restaurantes $-52 \%-$.

Entretanto, la informalidad laboral afecta indistintamente a hombres y mujeres: siete de cada diez mujeres mayores de 45 años, nueve de cada diez mujeres con solo educación básica primaria, y ocho de cada diez trabajadoras domésticas.

Para cerrar con estas cifras, el Boletín Mujeres en el Mercado Laboral Colombiano señala que tres de cada diez mujeres urbanas entre 18 y 28 años no estudian ni trabajan, y en mujeres rurales la relación es cinco de cada diez. Es importante destacar que estas mujeres pierden la oportunidad de educarse y desarrollarse laboralmente, lo cual las hace más vulnerables a condiciones de pobreza y en riesgo de exclusión laboral.

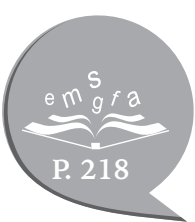


Por lo anterior, se plateó una investigación enfocada en conocer cómo las mujeres cabeza de familia que es una población con vulneración de sus derechos desarrollan actividades comerciales que les generen ingresos de manera independiente, haciendo uso de sus habilidades personales, que combinadas con educación y experiencia, reflejan mayores logros en el desarrollo de cualquier trabajo.

Para ello, se hizo un estudio de tipo descriptivo, caracterizando en la población situaciones y actitudes predominantes. La información se obtuvo por medio de una encuesta semiestructurada, aplicada a mujeres cabeza de hogar de estratos socioeconómicos uno y dos, a las cuales se les preguntó por sus ingresos económicos, formación profesional, actividad laboral, entre otros aspectos. El tipo de muestreo fue el determinístico aplicado a una muestra de 50 mujeres.

Las fases metodológicas iniciaron con la identificación del problema de investigación, la elección de las fuentes apropiadas, la selección, elaboración y aplicación de la herramienta de recolección de datos y el análisis y la interpretación de los datos recolectados.

Dentro de este contexto, Griffin y Van Fleet (2015) plantean un modelo de habilidades donde proponen que la combinación de educación, experiencia y habilidades personales conducen a evaluar las fortalezas y debilidades de cada persona autoevaluándose, conociéndose y visualizando las acciones propias y las de los demás, para así practicar, aplicar y extender las habilidades en diferentes contextos.

Los tres aspectos mencionados-educación, experiencia y habilidades- son claves en el análisis de las características de la población, ya que deja conocer si su combinación tiene efectos en la generación de ingresos para mejorar la economía del hogar.

Finalmente, la investigación arroja información concluyente en la que la combinación de educación, experiencia y habilidades personales genera unas habilidades gerenciales únicas que empoderan a las mujeres sujeto de estudio.

\section{Marco teórico}

\subsection{Habilidades gerenciales}

Diferentes autores han investigado sobre las habilidades gerenciales tras lo cual han coincidido que para gerenciar es indispensable contar con las habilidades propias de cada ser humano, teniendo en cuenta el sexo, la formación académica, el entorno social, la comunicación, el relacionamiento personal, el comportamiento y el buen juicio, entre otros aspectos. A continuación, se relacionan algunas teorías de autores que dan a conocer ampliamente el concepto de habilidades gerenciales.
De acuerdo con la teoría de Giddens (2010), las mujeres internalizan valores, normas y códigos distintos de los transmitidos a los hombres, los cuales reproducen patrones de comportamiento, que debido a la subvaloración de lo femenino generan desigualdades respecto de las habilidades necesarias para la gerencia, lo cual hace que las habilidades aprendidas y desarrolladas de manera diferente influyan -positiva o negativamente- en la acción emprendida y se moderen de manera desigual entre mujeres y hombres. 
Olaz (2011) plantea que las habilidades son elementos particulares de la personalidad de un individuo, que permiten la ejecución de tareas y determinar el desarrollo de una actividad o tarea.

Según Madrigal (2002), hay diferentes clases de habilidades que le permiten a un directivo lograr un mejor desempeño, tales como entender, desarrollar y aplicar habilidades interpersonales, sociales y de liderazgo, en tanto que las otras habilidades que se presentan son complementarias, lo cual llevará al directivo a contar con un perfil ideal para su desempeño. A continuación, se relacionan las habilidades directivas planteadas y su alcance (Tabla 1).

Tabla 1. Habilidades directivas

\begin{tabular}{|l|l|}
\hline Habilidades & Alcance \\
\hline Técnicas & Desarrollar tareas específicas. \\
\hline Interpersonales & $\begin{array}{l}\text { Se refiere a la habilidad para trabajar en grupo, con espíritu de cola- } \\
\text { boración, cortesía y cooperación para resolver las necesidades de otras } \\
\text { personas, incluso para obtener objetivos comunes. }\end{array}$ \\
\hline Sociales & $\begin{array}{l}\text { Son las acciones de uno con los demás y los demás con uno. Es donde } \\
\text { se da el intercambio y la convivencia humana. }\end{array}$ \\
\hline Académicas & $\begin{array}{l}\text { Capacidad y habilidad para hacer análisis, comparación, contratación, } \\
\text { evaluación, juicio o crítica. }\end{array}$ \\
\hline De innovación & $\begin{array}{l}\text { Invención, descubrimiento, suposición, formulación de hipótesis y teo- } \\
\text { rización. }\end{array}$ \\
\hline Prácticas & Aplicación, empleo e implementación -hábito-. \\
\hline Físicas & Autoeficiencia, flexibilidad y salud. \\
\hline De pensamiento & Aprender a pensar y generar conocimiento. \\
\hline Directivas & Saber dirigir y coordinar equipos de trabajo. \\
\hline De liderazgo & Guiar, impulsar y motivar al equipo hacia un bien común. \\
\hline Empresariales & Emprender una nueva idea, proyecto, empresa o negocio. \\
\hline
\end{tabular}

Fuente. Madrigal (2002).

Las acciones emprendidas por los seres humanos sin importar su finalidad son realizadas de acuerdo con las habilidades poseídas por cada uno, las cuales permiten que un individuo descubra oportunidades, persiga iniciativas que generen ingresos propios y procuren el desarrollo de una sociedad, entre otros. Las habilidades gerenciales reflejan actitudes de liderazgo, y en este sentido Jones y George (2014) identifican los rasgos y las características personales relacionados con un liderazgo eficaz ( Tabla 2). 
Tabla 2. Rasgos y características personales relacionados con un liderazgo eficaz

\begin{tabular}{|l|l|}
\hline \multicolumn{1}{|c|}{ Rasgo } & \multicolumn{1}{c|}{ Descripción } \\
\hline Inteligencia & $\begin{array}{l}\text { Ayuda a los gerentes a comprender asuntos complejos y resolver } \\
\text { problemas. }\end{array}$ \\
\hline Conocimiento y experiencia & $\begin{array}{l}\text { Ayudan a los gerentes a tomar buenas decisiones y describir formas } \\
\text { de mejorar la eficiencia y la eficacia. }\end{array}$ \\
\hline Dominio & $\begin{array}{l}\text { Ayuda a los gerentes a influir en sus subordinados para alcanzar las } \\
\text { metas organizacionales. }\end{array}$ \\
\hline Confianza en sí mismos & $\begin{array}{l}\text { Contribuye a que los gerentes influyan con eficacia en sus } \\
\text { subordinados y que persistan cuando enfrentan obstáculos difíciles. }\end{array}$ \\
\hline Energía alta & Ayuda los gerentes a atender las múltiples demandas que enfrentan. \\
\hline Tolerancia al estrés & $\begin{array}{l}\text { Permite a los gerentes enfrentar la incertidumbre y tomar decisiones } \\
\text { difíciles. }\end{array}$ \\
\hline Integridad y honestidad & $\begin{array}{l}\text { Ayudan a los gerentes a comportarse con ética y a ganarse la } \\
\text { confianza de sus subordinados. }\end{array}$ \\
\hline Madurez & $\begin{array}{l}\text { Ayuda a los gerentes a no actuar en forma egoísta, controlar sus } \\
\text { emociones y, en caso de cometer un error, a reconocerlo. }\end{array}$ \\
\hline
\end{tabular}

Fuente. Jones y George (2014).

Tres de las habilidades gerenciales comunes entre los autores mencionados son las relaciones interpersonales, el liderazgo y la toma de decisiones. Las relaciones interpersonales surgen mediante el establecimiento de una comunicación, la comprensión entre el emisor y el receptor y la retroalimentación del mensaje. Una comunicación adecuada permite que los objetivos de cualquier proyecto se cumplan; en este sentido, para las mujeres comerciantes cabeza de hogar, la comunicación es un factor clave de éxito, ya que ellas necesitan comunicarse constantemente con sus clientes para ofrecerles los nuevos productos que mensualmente salen al mercado y sus ofertas. Según Naranjo (2008), la comunicación guarda estrecha relación con la supervivencia de las personas; cuanta más información se conozca, más probabilidades de éxito se tendrá para una negociación.

La segunda habilidad por tener en cuenta es el liderazgo, que según Whetten y Cameron (2004), cada persona puede desarrollar las habilidades necesarias para liderar el cambio; nadie nació como líder ni sin las habilidades que lo podrían facultar como tal, por lo que todos los individuos pueden ser líderes en algún momento. Las mujeres sujeto de la investigación no han necesitado formación profesional para ser líderes en sus actividades laborales, no han realizado cursos de liderazgo que las hayan llevado a asumir actitudes de liderazgo en sus actuaciones. A las mujeres cabeza de familia les ha tocado asumir ese papel, en muchos casos, debido al machismo o cultura patriarcal, lo cual tiene repercusiones en su vida, como exclusión de oportunidades de estudio y trabajo. Estos dos aspectos influyen para que no mejoren sus condiciones de vida, nivel de ingresos o tipo de trabajo que desarrollan.

La otra habilidad es la toma de decisiones, que, de acuerdo con Gómez y Balkin (2010), es el proceso de identificar las oportunidades, los problemas y resolverlos. Las decisiones tomadas por las mujeres comerciantes cabeza de hogar empiezan al identificar y diagnosticar 
un problema u oportunidad desde el momento en que están ofreciendo los productos hasta el relacionamiento que mantienen con los clientes después de la venta, lo cual las lleva a tomar decisiones de mejora en sus ventas, estrategias de comercialización y de relacionamiento, hecho que se convierte en un ciclo permanente. La toma de decisiones no siempre es asertiva si no se tiene la información completa del contexto y si no se parte de la raíz del problema. Gómez y Balkin plantean que la toma de decisiones se divide en seis etapas: i) identificación del problema; ii) generación de soluciones alternativas; iii) evaluación alternativa; iv) selección de la mejor alternativa, v) implantación de la decisión; y vi) evaluación de la decisión.

Jones y George (2014) afirman que la educación y la experiencia permiten a los gerentes reconocer y desarrollar tres tipos de habilidades: conceptuales, humanas y técnicas, para desempeñarse de la mejor manera, refiriéndose a las primeras como la capacidad para analizar y diagnosticar una situación, a las segundas como la capacidad para entender, modificar, dirigir o controlar la conducta de otros individuos y a las terceras como las requeridas para desempeñar un tipo particular de trabajo u ocupación.

\subsection{Educación, ocupación, desempleo e ingresos en los hogares}

De acuerdo con ONU Mujeres Colombia (s. f.), en educación, se ha logrado un gran progreso. Las mujeres colombianas tienen una tasa más alta de educación que los hombres. Sin embargo, las mujeres aún enfrentan importantes dificultades de acceso al empleo, y cuando entran en el mercado laboral, se enfrentan a diferencias salariales significativas y trabajan en los altos niveles de informalidad.

Se muestra a nivel nacional para 2016 la tasa de desempleo según el nivel educativo logrado y el sexo, en la cual se evidencia que la mayor tasa de desempleo se registra en las mujeres sin importar su nivel educativo (Figura 1). De esta forma, el porcentaje de desempleo de las mujeres que tiene formación en educación media -grado once- se encuentra en el $14.8 \%$, a diferencia del porcentaje de desempleo de los hombres que es del $8.9 \%$ con el mismo nivel educativo; las mujeres sujeto de estudio, al verse sin oportunidades de empleo, se ven en la necesidad de buscar a como dé lugar ingresos que cubran sus necesidades básicas de alimentación, salud y vivienda para ellas y sus familias. Esto tiene efectos en su vida y grupos familiares, ya que son los que más niveles de pobreza pueden registrar. 
Figura 1. Tasa de desempleo según nivel educativo logrado y sexo -total nacional 2016-

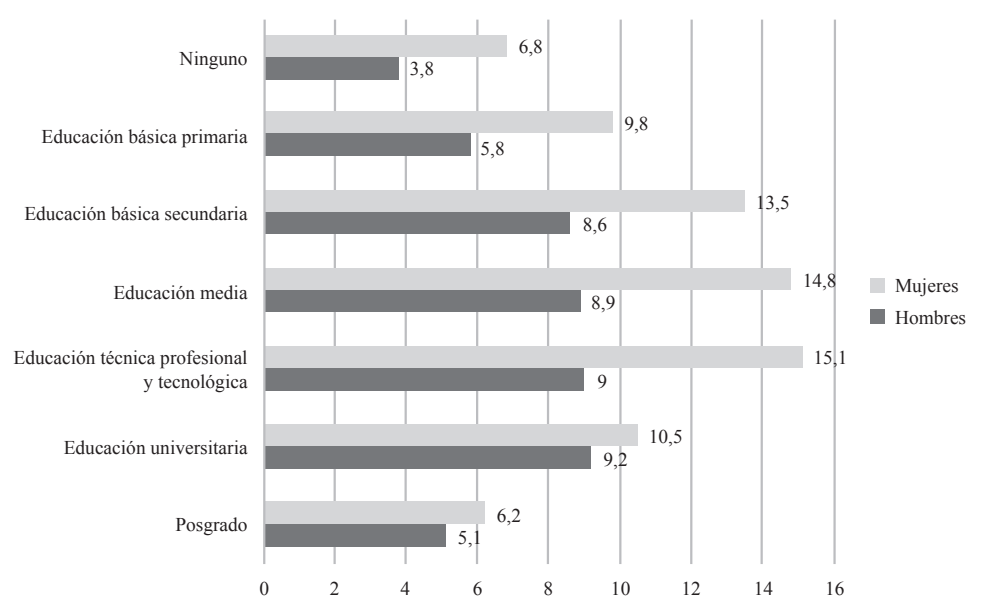

Fuente. Departamento Administrativo Nacional de Estadística (DANE, s. f.).

Se presenta la distribución porcentual de ocupados según su posición ocupacional en 2016 como empleados particulares, empleados del Gobierno, empleados domésticos, trabajadores por cuenta propia, empleadores, trabajadores sin remuneración (Figura 2). Así, los mayores ocupados obedecen a los trabajadores por cuenta propia que buscan en su entorno actividades laborales que les permitan conseguir ingresos sin tener ningún nivel de estudios, los empleados que le siguen con mayor porcentaje de ocupación laboral son los obreros o empleados particulares que cuentan con educación técnica, profesional o tecnológica, los empleados del Gobierno que tienen mayor ocupación son los que poseen educación universitaria o posgrado y un empleado doméstico no requiere mucha formación para ser contratado. Por su parte, los empleados domésticos más ocupados son los que poseen educación básica primaria; los porcentajes en los niveles de educación para un patrón o empleador no varían mucho, un patrón o empleador puede no tener ninguna formación o puede poseer posgrado, rangos que se encuentran entre el 3.3 y $5.5 \%$.

Figura 2. Distribución porcentual de ocupados según nivel educativo logrado y posición ocupacional -total nacional 2016-

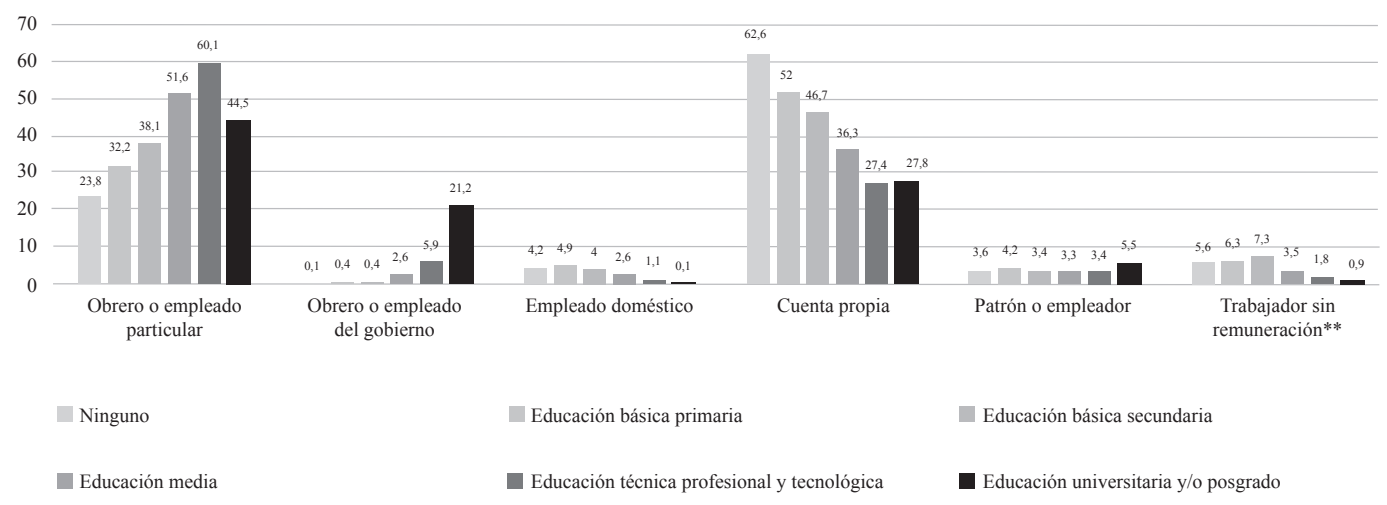

Fuente. DANE (s. f.). 
Por otro lado, se registran los porcentajes de los ingresos recibidos teniendo en cuenta la formación académica de la población colombiana en 2016 (Figura 3). El mayor ingreso -más de 1.5 SMMLV-, se ve en el porcentaje de la población que tiene educación universitaria o posgrado -79.9\%-; el $18.8 \%$ de las personas que no tuvienon ninguna formación se ganan entre $1 \mathrm{SMMLV}$ y 1.5 SMMLV, mientras que el $11.4 \%$ con formación profesional ganan lo mismo, presentándose que en este rango hay más oporunidades para los menos estudiados.
La figura de distribución porcentual refleja que a mayor ingreso mayor es la necesidad de formación y a menor salario menor es el nivel educativo logrado. Las mujeres cabeza de hogar encuestadas se ubican en la población que recibe ingresos entre 0.5 y 1 SMMLV y en los de 1 y 1.5 SMMLV. Con estos ingresos, no cubren las necesidades básicas de familias de más de cuatro personas; por eso, ellas buscan aumentar sus ingresos mensuales aplicando habilidades interpersonales, en la promoción de nuevos servicios o productos que satisfagan las necesidades de su grupo familiar.

Figura 3. Distribución porcentual de ocupados según nivel educativo logrado y rangos de ingreso -total nacional 2016

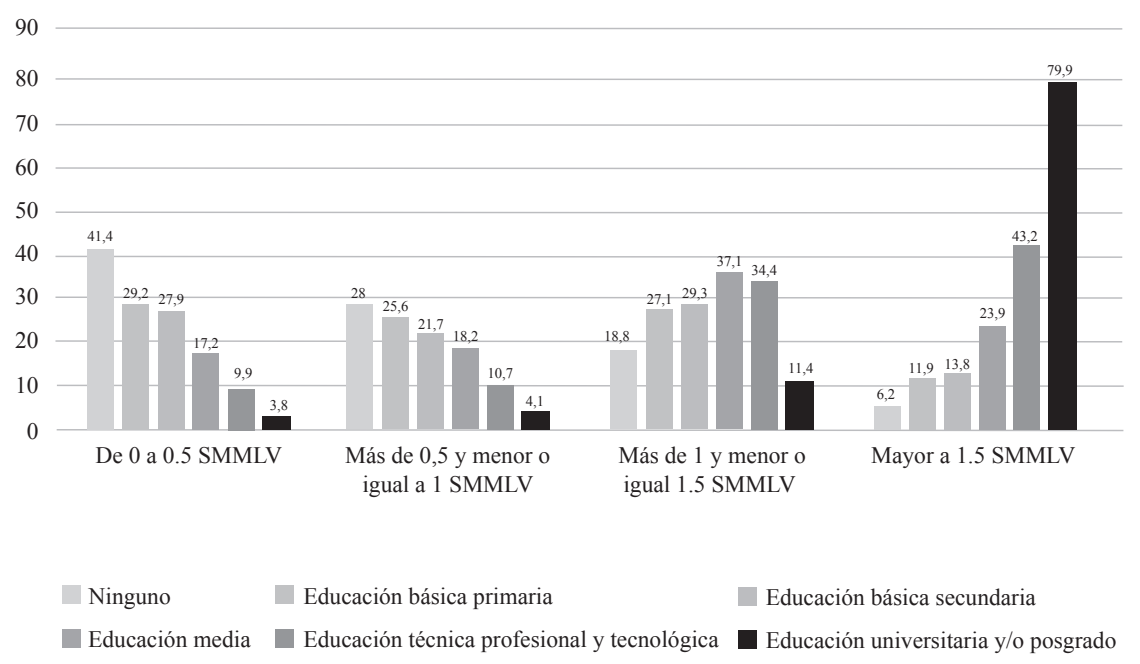

Fuente. DANE (s. f.). 


\section{Metodología}

E sta es una investigación de tipo descriptivo, que caracteriza la población sujeto de estudio partiendo de la identificación del problema de investigación, para luego elegir las fuentes apropiadas para ella. Esta es de aproximadamente 1200 mujeres cabeza de hogar que tienen bajos niveles de ingresos

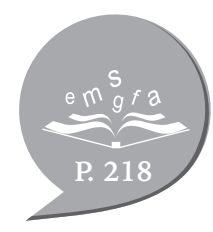
en Montería, Colombia y su casco urbano. De esta población se tomó una muestra por conveniencia de 50 mujeres cabeza de hogar dedicadas a la

comercialización de productos por catálogo, suscritas $\sin$ experiencia y sin formación profesional en empresas nacionales e internacionales para la distribución de sus productos. Se seleccionaron por la facilidad de contacto con ellas y por los bajos costos de desplazamiento para aplicación del instrumento. Finalmente, se realizó el tratamiento, la tabulación, el análisis y la interpretación de los datos recolectados para la elaboración de las conclusiones del estudio.

\section{Análisis de resultados}

$\mathrm{Z}^{1}$ grupo de personas encuestadas se Eencuentra entre los 29 y 70 años, quienes culminaron su educación básica hasta noveno grado- en un $35 \%$ y su educación media -décimo y onceavo- en un $40 \%$. Por otro lado, el $20 \%$ terminó una carrera técnica; y el restante $5 \%$, una carrera tecnológica. Por lo anterior, se infiere que el nivel educativo de la mayoría de las encuestadas es básico. Como dato importante, la educación no es un requisito para que las vendedoras o consultoras de las empresas de productos de belleza por catálogo se vinculen.

La población encuestada en un $40 \%$ tiene a su cargo dos personas como hijos, esposo sin trabajo o padres, un $20 \% 3$ personas, el otro $20 \% 4$ personas, el resto de las encuestadas no tienen a cargo a los miembros de su grupo familiar, pero con sus ventas contribuyen en gran proporción a la economía del hogar, además de asumir sus gastos personales.

Los ingresos mensuales oscilan entre COP 200000 y COP 800 000, valores bajos para cubrir necesidades básicas, ya que sus grupos familiares están conformados por más de cuatro personas. Las mujeres que más ingresos reciben son aquellas que comercializan diferentes artículos como prendas de vestir, calzado y algunos electrodomésticos. Laboran en empresas como Avon, L'Bel, Natura, Leonisa, Aquarella, Marketing Personal, entre otras, las cuales les ofrecen como consultoras un $25 \%$ de ganancia mensual si venden desde COP 210000 hasta COP 480000 en productos, un $30 \%$ de ganancia si venden entre COP 481000 y COP 1200 000, un $35 \%$ de ganancia si venden entre COP 1201000 y COP 1600000 y un $40 \%$ de ganancia si venden más de COP 1600000.

Las empresas líderes en ventas por catálogo tienen diferentes clasificaciones para sus consultoras, que van aumentando de acuerdo con sus ventas; inician con la consultora nueva y finalizan con la empresaria brillante, que es la que más ingresos por ventas recibe. Para asegurarse de mantenerlas motivadas les dan cada dos meses obsequios como electrodomésticos, maquillaje, estuches, entre otros artículos, que ellas utilizan para su uso personal o para venderlos. Además de los regalos, la motivación 
la incrementan realizando charlas mensuales y capacitaciones cada tres meses en las cuales les enseñan a probar los productos que están vendiendo, conseguir clientes, entregar su propuesta de valor, relacionarse, realizar los pedidos de manera virtual -algo que para las personas mayores de 50 años vinculadas a las empresas no ha sido fácil por su desconocimiento en el uso de herramientas tecnológicas-, pagar sus facturas y continuar el ciclo de venta de las consultoras vendiendo mensualmente.

En vista de que hay situaciones donde las consultoras necesitan capital para movilizarse a ofrecer los productos, entregarlos, realizar visitas a posibles clientes, muchas se desmotivan y buscan otras fuentes de generación de ingresos relacionadas con las ventas, por lo cual las empresas constantemente le enseñan a planear sus metas, organizar bien sus pedidos, controlar sus ingresos mensuales y cumplir con sus objetivos manteniendo su motivación.

En cuanto a la formación que reciben las mujeres cabeza de hogar en las empresas nacionales e internacionales de ventas de productos por catálogo en las cuales se encuentran afiliadas, tienen una metodología de formación basada en el modelo de negocios Canvas planteado por Osterwalder y Pigneur (2013), donde ellas inician su labor teniendo claro cuál es la oferta de valor que tienen los productos que están comercializando, identificando su segmento de clientes en el mercado, formulando estrategias para el relacionamiento con los clientes, reconociendo las actividades clave, buscando aliados clave y calculando los ingresos y costos generados en la comercialización de los productos. De este modelo se desprende otro tipo de capacitaciones que les permiten aprender acerca de servicio al cliente, marketing personal, finanzas personales, planeación estratégica, para finalmente cumplir sus metas tanto personales como laborales.
Con lo anterior, las consultoras adquieren habilidades conceptuales donde tienen la capacidad de diagnosticar situaciones y saber qué decisiones tomar cuando se les presenten dificultades u oportunidades en el medio. Si bien es cierto que las habilidades conceptuales se adquieren mediante la educación formal y las capacitaciones (Jones y George, 2014), las mujeres cabeza de hogar que se dedican a la comercialización de productos han adquirido estas habilidades gracias a su perseverancia en su actividad comercial a lo largo de los años, ya que el $80 \%$ de las mujeres encuestadas llevan entre 11 y 15 años en la misma actividad, y a los estímulos que les brindan las empresas nacionales e internacionales. El $50 \%$ de las encuestadas expresan que para vender es necesaria la influencia personal de cada una de ellas, por lo cual la planeación es un factor clave en sus actividades diarias; el $33.3 \%$ expresa que la organización les ayuda igualmente a programar sus actividades, optimizar el tiempo y a la vez el dinero y el $16.7 \%$ señala que, si no existe control y seguimiento en ventas y en los ingresos mensuales, no podrían cumplir sus metas.

Al mismo tiempo adquieren habilidades humanas como el liderazgo, el servicio al cliente, la comunicación interpersonal y el conocimiento de las necesidades de sus clientes; realizan negociaciones de manera ética teniendo en cuenta su buen juicio; y aprenden a administrar bien su tiempo. De acuerdo con la investigación realizada, el $66.7 \%$ de la población mantiene en alto su ética, ya que de eso depende sus futuras negociaciones, le siguen con un $33.3 \%$ las habilidades comunicativas, que son habilidades para relacionarse con los clientes que se convierten en personas confiables, sinceras y con agilidad mental, su mente las hace personas expertas en habilidades sociales lo cual les permite tener mayor confianza en sí 
mismas, ser tolerantes, con dominio propio, maduras, personas que escuchan, inician una conversación, dan las gracias, se disculpan e innovan al solucionar problemas. Aquí se identifican rasgos y características relacionados con un liderazgo eficaz planteado por Jones y George (2014).

Sumado a lo anterior, la población también desarrolla habilidades técnicas las cuales se relacionan con la capacidad de poder utilizar a su favor los recursos necesarios para realizar tareas específicas. Para ello, se requiere tener dominio de una carrera o una práctica profesional (Madrigal, 2002); en este caso, no se da el dominio de una carrera profesional, pero sí la práctica de la actividad comercial. Un $83.3 \%$ de las personas encuestadas expresan que cuentan con conocimientos en mercadeo porque las ventas es lo que realizan diariamente y con apoyo de capacitaciones recibidas saben aplicar estrategias que mantienen a estas mujeres adquiriendo siempre nuevos clientes. Por su parte, el $16.7 \%$ expresa que cuenta con conocimientos en el área contable, lo cual les permite mantener sus cuentas al día, por esta razón desarrollan no solo habilidades de mercadeo sino también financieras.

Dentro de este contexto, el $83.3 \%$ de las encuestadas logran satisfacer sus necesidades primarias de salud, vivienda, educación y alimentación con los ingresos mensuales recibidos; y el $16.7 \%$ restante, además de sus necesidades primarias, las necesidades secundarias que consisten en recreación, cultura y movilización.

\section{Conclusiones}

T uego del acercamiento y el proceso de Lanálisis de los datos recolectados, se concluyó que a través del ejercicio de su labor como consultoras, las mujeres sujeto de estudio logran desarrollar una fuerte experiencia en ventas que se afianza con las capacitaciones que les brindan las compañías, con una frecuencia mínima de cada seis meses. Cuando se da una sinergia entre estos dos procesos, ellas logran desarrollar sus habilidades gerenciales y son capaces de dirigir su propio trabajo y se empoderan de su rol de consultoras hasta el punto de que se sienten dueñas de su negocio; muchas no perciben que están trabajando para otra compañía sino para ellas mismas.
Muchas de las mujeres encuestadas también asisten a capacitaciones de programas del Gobierno, enfocadas en la promoción de la igualdad de género y en el empoderamiento de las mujeres, como lo es Casa de la Mujer; por tanto, ellas son conscientes y se sienten motivadas a buscar su empoderamiento que les permita lograr una equidad de género. Esto lo hacen indagando o lo conocen por el voz a voz de colegas, amigos o familiares.

Por tanto, logran armonizar cada uno de los procesos del negocio, que va desde la promoción, la demostración, la fidelización, la toma de pedido, la entrega de pedido y el recaudo de cartera, lo cual se ve reflejado en el crecimiento como consultoras hasta lograr escalar niveles superiores del negocio y aumentar sus porcentajes de rentabilidad. 
Sin embargo, aunque las consultoras cuentan con herramientas básicas para escalar en su oficio de consultoras de ventas, es difícil para ellas llegar a la más alta categoría de vendedoras empresaria brillante a causa de su rol de mujeres cabeza de hogar, que les permite solo dedicarse a sus actividades comerciales en forma parcial, medio tiempo o un cuarto de tiempo diariamente.

Por lo anterior, las mujeres encuestadas hallan varios obstáculos financieros, de tiempo y de conocimiento que no los solucionan por sí solas: el $50 \%$ encuentra apoyo en su familia, si los obstáculos son económicos el $25 \%$ acude a los pagadiarios y el otro $25 \%$ acude a entidades de financiamiento comercial.

Por otro lado, el hecho de que solo el $25 \%$ haya realizado estudios técnicos o tecnológicos no ha sido una limitante para que el resto de las mujeres sujeto de estudio buscaran una alternativa de consecución de ingresos y convirtieran el ejercicio de ventas directas en la oportunidad para hacerse económicamente activas, lograr un crecimiento económico y experimentar un progreso social, que se traduce en empoderamiento de las mujeres, progreso humano para ellas y sus familias.

$\mathrm{Si}$ bien los ingresos que reciben no superan los 2 SMMLV en Colombia, el $83.3 \%$ se mantiene en un estrato 1,2 y 3 satisfaciendo sus necesidades básicas o primarias de alimentación, salud, vivienda y educación; el $16.7 \%$ restante supera las metas y así logra satisfacer las necesidades de segundo orden relacionadas con el turismo, los vehículos y la cultura, lo que las lleva a esforzarse y motivarse cada día más porque ven oportunidades para hacerlo con la ayuda de empresas que las incentivan y capacitan para ser cada día mejores en su oficio como consultoras de venta.

Las mujeres cabeza de hogar se capacitan por lo menos cada seis meses, con las charlas o capacitaciones que brindan las empresas con las que laboran o con programas del Gobierno como Casa de la Mujer, cada una de ellas se siente motivada con lo que realizan y siempre están buscando aumentar sus ventas mes a mes porque de ello depende sus ingresos.

Clasificando las habilidades gerenciales en habilidades conceptuales, técnicas, humanas, interpersonales, de liderazgo y de toma de decisiones, estas son desarrolladas básicamente por su experiencia, la experiencia de sus colegas y las capacitaciones recibidas; sus mayores habilidades se encuentran en la ética, la administración del tiempo, el relacionamiento con el cliente, el logro de sus metas, la agilidad mental y el entendimiento al cliente; y su mayor habilidad en cuanto a temas conceptuales se encuentra en mercadeo y contabilidad.

Finalmente, se concluye que las mujeres comerciantes cabeza de hogar necesitan mantenerse capacitadas en estrategias de mercadeo, en servicio al cliente, en la utilización de herramientas tecnológicas, en el manejo contable, en el manejo de clientes y en el liderazgo, entre otros temas gerenciales, para poder mantenerse como buenas vendedoras, satisfacer las necesidades de sus clientes, aumentar sus ingresos, superarse cada día y aportar en el desarrollo económico y social de la región. 


\section{Referencias}

Alcaldía de Montería (2012). Plan Educativo Rural 2012-2015. Recuperado de http://aprende.colombiaaprende.edu.co/ckfinder/userfiles/files/ PER \%20MONTERIA \%202012.pdf

Cárdenas Rubio, J. A. (2013). El impacto de la participación femenina en el mercado laboral de los hombres en Colombia desde 1984 hasta 2012. Recuperado de http://repository.urosario.edu.co/bitstream/handle/10336/4794/1098665552-2013.pdf? sequen$\mathrm{ce}=1$

Departamento Administrativo Nacional de Estadística (2017). Fuerza laboral y educación. Recuperado de https://www.dane.gov.co/files/investigaciones/boletines/especiales/educacion/Bol_edu_2016.pdf

Departamento Administrativo Nacional de Estadística (s. f.). Gran encuesta integrada de hogares - GEIHmercado laboral. Recuperado de https://www.dane. gov.co/index.php/estadisticas-por-tema/mercado-laboral/empleo-y-desempleo\#2016

Giddens, A. (2010). Sociología. Madrid: Alianza Editorial.

Gómez-Mejía, L. R. y Balkin, D. B. (2010). Administración. México: McGraw-Hill.

Griffin, R. y Fleet, D. van (2015). Habilidades directivas: evaluación y desarrollo. México: Cengage.
Jones, G. R. y George, J. M. (2014). Administración contemporánea. México: McGraw-Hill.

Madrigal Torres, B. E. (2002). Habilidades directivas. México: McGraw-Hill.

Naranjo Arango, R. (2008). Habilidades del líder. Cali: Lithoclave.

Observatorio Servicio Público de Empleo (2016). Boletín Mujeres en el Mercado Laboral Colombiano. Recuperado de http://serviciodeempleo.gov.co/observatorio/2016/08/17/boletin-mujeres-en-el-mercado-laboral-colombiano/

Olaz, Á. (2011). Una aproximación conceptual a la cualificación profesional desde una perspectiva competencial. Papers: Revista de Sociología, 96(2), 589-616.

ONU Mujeres Colombia (s. f.). Las mujeres en Colombia. Recuperado de http://colombia.unwomen.org/es/ onu-mujeres-en-colombia/las-mujeres-en-colombia

Osterwalder, A. y Pigneur, Y. (2010). Business model generation: A handbook for visionaries, game changers, and challengers. New Jersey: John Wiley \& Sons.

Whetten, D. A. y Cameron, K. S. (2004). Desarrollo de habilidades directivas. México: Pearson. 